Ferrata Storti Foundation

\title{
CCR5 antagonism by maraviroc inhibits Hodgkin lymphoma microenvironment interactions and xenograft growth
}

\author{
Naike Casagrande,${ }^{1}$ Cinzia Borghese,${ }^{1}$ Lydia Visser, ${ }^{2}$ Maurizio Mongiat, ${ }^{1}$ \\ Alfonso Colombatti ${ }^{1}$ and Donatella Aldinucci ${ }^{1}$
}

${ }^{1}$ Unit of Molecular Oncology, Centro di Riferimento Oncologico di Aviano (CRO) IRCCS, Italy and ${ }^{2}$ Department of Pathology and Medical Biology, University Medical Center Groningen (UMcG), the Netherlands

Haematologica 2019

Volume 104(3):564-575

NC and CB contributed equally to this study.

\section{Correspondence:}

DONATELLA ALDINUCCI

daldinucci@cro.it

Received: May 3, 2018.

Accepted: October 9, 2018

Pre-published: October 11, 2018.

doi:10.3324/haematol.2018.196725

Check the online version for the most updated information on this article, online supplements, and information on authorship \& disclosures: www.haematologica.org/content/104/3/564

\section{(C)2019 Ferrata Storti Foundation}

Material published in Haematologica is covered by copyright. All rights are reserved to the Ferrata Storti Foundation. Use of published material is allowed under the following terms and conditions:

https://creativecommons.org/licenses/by-nc/4.0/legalcode. Copies of published material are allowed for personal or internal use. Sharing published material for non-commercial purposes is subject to the following conditions:

https://creativecommons.org/licenses/by-nc/4.0/legalcode, sect. 3. Reproducing and sharing published material for commercial purposes is not allowed without permission in writing from the publisher.

\section{(c) (i) (5)} \\ ABSTRACT}

lassic Hodgkin lymphoma tumor cells express a functional CCR5
receptor, and tumor tissues express high CCL5 levels, suggesting
that CCL5-CCR5 signaling is involved in tumor-microenvironment formation and tumor growth. Using the CCR5 antagonist, maraviroc, and a neutralizing anti-CCL5 antibody, we found that CCL5 secreted by classic Hodgkin lymphoma cells recruited mesenchymal stromal cells and monocytes. The "education" of mesenchymal stromal cells by tumor cell-conditioned medium enhanced mesenchymal stromal cells' proliferation and CCL5 secretion. In turn, educated mesenchymal stromal cell-conditioned medium increased the clonogenic growth of tumor cells and monocyte migration, but these effects were reduced by maraviroc. Monocyte education by tumor cell-conditioned medium induced their growth and reprogrammed them towards immunosuppressive tumor-associated macrophages that expressed IDO and PD-L1 and secreted IL-10, CCL17 and TGF- $\beta$. Educated monocyte-conditioned medium slowed the growth of phytohemagglutinin-activated lymphocytes. Maraviroc decreased tumor cell growth and synergized with doxorubicin and brentuximab vedotin. A three-dimensional heterospheroid assay showed that maraviroc counteracted both the formation and viability of heterospheroids generated by co-cultivation of tumor cells with mesenchymal stromal cells and monocytes. In mice bearing tumor cell xenografts, maraviroc reduced tumor growth by more than $50 \%$ and inhibited monocyte accumulation, without weight loss. Finally, in classic Hodgkin lymphoma human tumor tissues, CCL5 and CD68 expression correlated positively, and patients with high CCL5 levels had poor prognosis. In conclusion, since the present challenges are to find molecules counteracting the formation of the immunosuppressive tumor microenvironment or new, less toxic drug combinations, the repurposed drug maraviroc may represent a new opportunity for classic Hodgkin lymphoma treatment.

\section{Introduction}

Inflammatory chemokines are indispensable "gate-keepers" of inflammation and immunity against cancer, but tumor cells can subvert chemokines into acting as tumor-promoting molecules. ${ }^{1} \mathrm{C}$-C motif chemokine ligand 5 (CCL5) is one such chemokine that can favor tumor development in multiple ways; for example, by acting as a growth factor for tumor cells, stimulating angiogenesis, recruiting stromal and inflammatory cells, and taking part in immune evasion mechanisms. ${ }^{2-6}$ CCL5 belongs to the C-C chemokine family whose members include CCL3 and CCL4. ${ }^{1,2}$ Its activity is mediated through binding to CCR1, CCR3, and CCR5, while CD44 serves as an auxiliary receptor. ${ }^{2}$

CCL5 and other chemokines are expressed at higher levels in classic Hodgkin lymphoma (cHL) tumor tissues than in healthy lymph nodes and in tissues with 
reactive lymphoid hyperplasia., ${ }^{7,8}$ Both CCL5 and its receptor CCR 5 are constitutively expressed by cHL-derived cell lines ${ }^{7}$ by tumor cells from cHL lymph node tissues and by bystander cells including stromal cells and lymphocytes.? The CCR5 receptor expressed by cHL cells is fully functional and its ligands function as both paracrine and autocrine growth factors.

The interactions of cHL tumor cells with a variety of non-tumor reactive cells accumulating in $\mathrm{cHL}$ tissues mediate tumor cell growth, formation of an immunosuppressive, protective tumor microenvironment (TME), neoangiogenesis, ${ }^{9}$ and drug resistance. ${ }^{10,11}$ Increasing evidence suggests that not only $\mathrm{T}$ cells, ${ }^{12}$ but also mesenchymal stromal cells (MSCs) ${ }^{13}$ and monocytes, ${ }^{14,15}$ contribute to the TME in cHL. ${ }^{11,16}$ MSCs, by modulating NKG2D expression in $\mathrm{T}$ cells and its ligand in tumor cells, reduce the immune response against $\mathrm{cHL}$ cells. ${ }^{13} \mathrm{~A}$ high number of infiltrating macrophages, ${ }^{17,18}$ predominantly derived from circulating monocytes, ${ }^{19}$ and a high absolute monocyte count in peripheral blood both correlate with poor cHL prognosis. ${ }^{20,21}$ These observations likely reflect the ability of $\mathrm{cHL}$ cells to reprogram macrophages towards immunosuppressive tumor-associated macrophages (TAMs). ${ }^{20,21}$

Given current knowledge about cell-cell interactions in $\mathrm{cHL}$, there is interest in drugs that can interfere with this crosstalk. ${ }^{22-25}$ But since drug discovery is expensive and time-consuming, drug repurposing is an attractive approach for finding new cancer treatments. ${ }^{26}$ One such repurposed drug is the CCR5 antagonist maraviroc. ${ }^{27}$ Approved by the US Food and Drug Administration for the treatment of HIV infection, maraviroc causes few side effects in humans, even during long-term therapy. ${ }^{28,29}$ As an anticancer drug, maraviroc has different effects: it blocks metastasis of basal breast cancer cells; ${ }^{30}$ it decreases the migration of regulatory $T$ cells; it reduces metastatic breast cancer growth in the lungs; ${ }^{31}$ and it inhibits the accumulation of fibroblasts in human colorectal cancer. ${ }^{32}$ Maraviroc reprograms immunosuppressive myeloid cells and reinvigorates antitumor immunity by targeting the autocrine CCL5-CCR5 axis in bone marrow. ${ }^{6}$ It also polarizes macrophages towards an M1-like functional state. ${ }^{27}$

Our working hypothesis is that cHL cancer cells, by secreting CCL5, recruit both MSCs and monocytes to the TME, and then reprogram these cell types to make them pro-tumorigenic. Thus, blocking the CCR5 receptor should inhibit not only tumor growth, as we previously observed, but also the recruitment of cells to form the protective, immunosuppressive TME.

Here, we investigated the role of CCL5-CCR5 signaling in the interactions of monocytes and MSCs with cHL cells, using, in particular, three-dimensional multicellular heterospheroids ${ }^{33}$ formed by tumor cells, monocytes and MSCs, as well as an in vivo cHL model and tissues from cHL patients.

\section{Methods}

Maraviroc (Sigma-Aldrich) was dissolved in DMSO at $51.8 \mathrm{mM}$. Other reagents are detailed in Online Supplementary Methods, together with protocols for cell migration, proliferation, clonogenic growth and senescence assays, immunosuppression, synergy, flow cytometry, ELISAs and other cell-based assays, immunofluorescence, survival of tumor xenografts and statistical analysis.

\section{Cell culture and conditioned media}

Authenticated cHL-derived cell lines L-1236, L-428, KMH2, HDLM-2, and L-540 (DSMZ, Germany) were cultured in RPMI-1640 medium containing 10\% fetal calf serum (FCS). To prepare conditioned medium (CM) from $\mathrm{cHL}$ cell lines, cells were seeded at $2.0 \times 10^{5} / \mathrm{ml}$ in RPMI-1640 plus $10 \%$ FCS, and the medium was collected after $72 \mathrm{~h}$. Human bone marrow-derived and adipose tissue-derived mesenchymal stromal cells (MSCs) (BM-MSCs and ATMSC, respectively) were purchased from Lonza (Verviers, Belgium). cHL-MSCs from frozen lymph nodes were generated as described in Online Supplementary Methods. BMMSCs, AT-MSC and cHL-MSCs were maintained in Mesenchymal Stem Cell Growth Medium Bulletkit (Lonza) to avoid differentiation.

Monocytes were isolated from peripheral blood mononuclear cells (PBMCs) from healthy donor blood using CD14 Microbeads, Human (Miltenyi Biotec). To generate tumor-educated MSCs (E-MSCs) and monocytes (E-monocytes), MSCs and monocytes were cultured separately for 6 days in complete culture medium (RPMI-1640, $10 \%$ FCS) containing 20\% CM from cHL cell lines; half the volume of medium was replaced every other day. To prepare CM from these tumor-educated cells, they were washed and cultured in fresh medium for $72 \mathrm{~h}$.

\section{D culture of heterospheroids}

Heterospheroids were generated by co-culturing various combinations of cHL cells, HL-MSCs and monocytes (1.0 $\times 10^{4} / \mathrm{mL}$ of each cell type) in RPMI-1640 medium containing $1 \%$ FCS, using plates coated with $20 \mathrm{mg} / \mathrm{mL}$ polyHEMA (Sigma) to prevent adhesion. After 4 days, heterospheroids were tested for CCL5 secretion into the medium by ELISA. In some experiments, heterospheroids were treated with maraviroc, alone or with doxorubicin, for 6 days. Growth was evaluated using the PrestoBlue Cell Viability Reagent (Invitrogen).

\section{Tumor xenograft experiments}

Animal experiments were approved by the Italian Ministry of Health (no. 671/2015/PR). We used ten 4week-old female athymic nude/nude mice (Harlan Laboratories) and ten 4-week-old male NOD/SCID gamma chain deficient (NSG) mice (Charles River). L-540 $\left(200 \times 10^{6}\right.$ cells/animal $)$ and $\mathrm{L}-428$ cells $\left(10 \times 10^{6}\right.$ cells/animal $)$ were suspended in Matrigel (diluted 1:3 in PBS) and inoculated into the flank of nude mice (L-540) or NSG mice (L-428). When tumors were palpable, animals were divided into two equal groups and treated every day (L-540) or every other day (L-428) with maraviroc (intraperitoneal injection, $10 \mathrm{mg} / \mathrm{kg})^{34}$ or vehicle (PBS). Body weight and tumor volume were measured daily.

\section{Immunohistochemistry tissue microarray analysis of cHL patients}

The study protocol was approved by the institutional review board of the University Medical Center Groningen. We recruited 65 patients with cHL (Online Supplementary Table S1). All study subjects provided written informed consent. Immunohistochemistry was performed for CCL5 (C-19 antibody, 1:200 dilution, Santa Cruz Biotechnology) (antigen retrieval in $10 \mathrm{mM}$ Tris-HCl $\mathrm{pH}$ 9, 1 mM EDTA). CD68 was detected with KP1 antibody (1:4000 dilution, Dako) (antigen retrieval in $10 \mathrm{mM}$ citrate buffer, $\mathrm{pH}$ 6). 

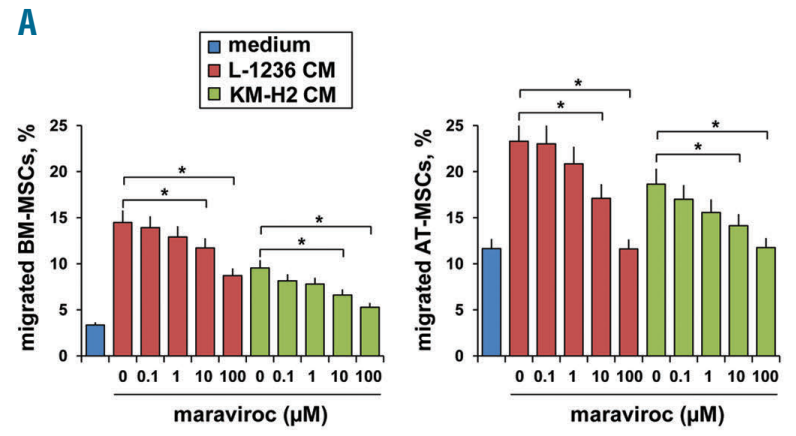

C

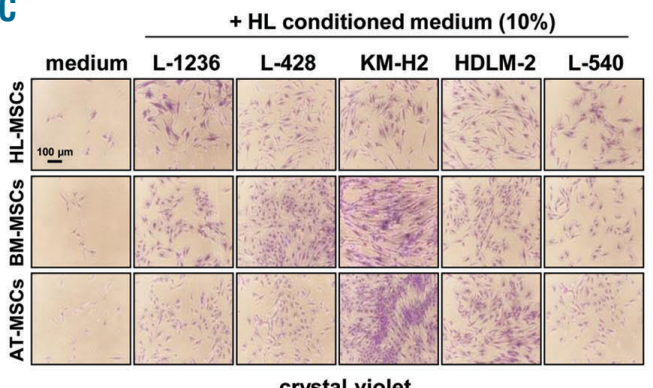

D

crystal violet
B

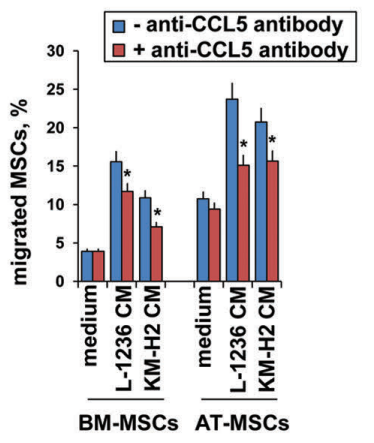

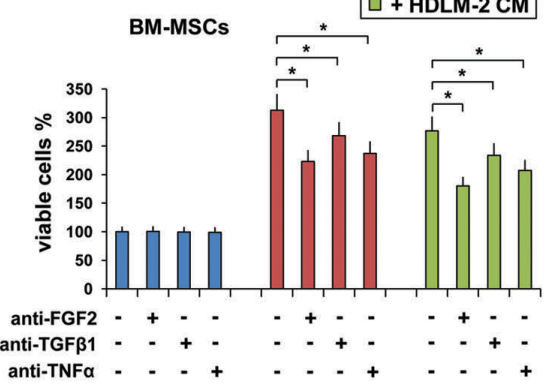

$\mathrm{E}$

BM-MSCs CM
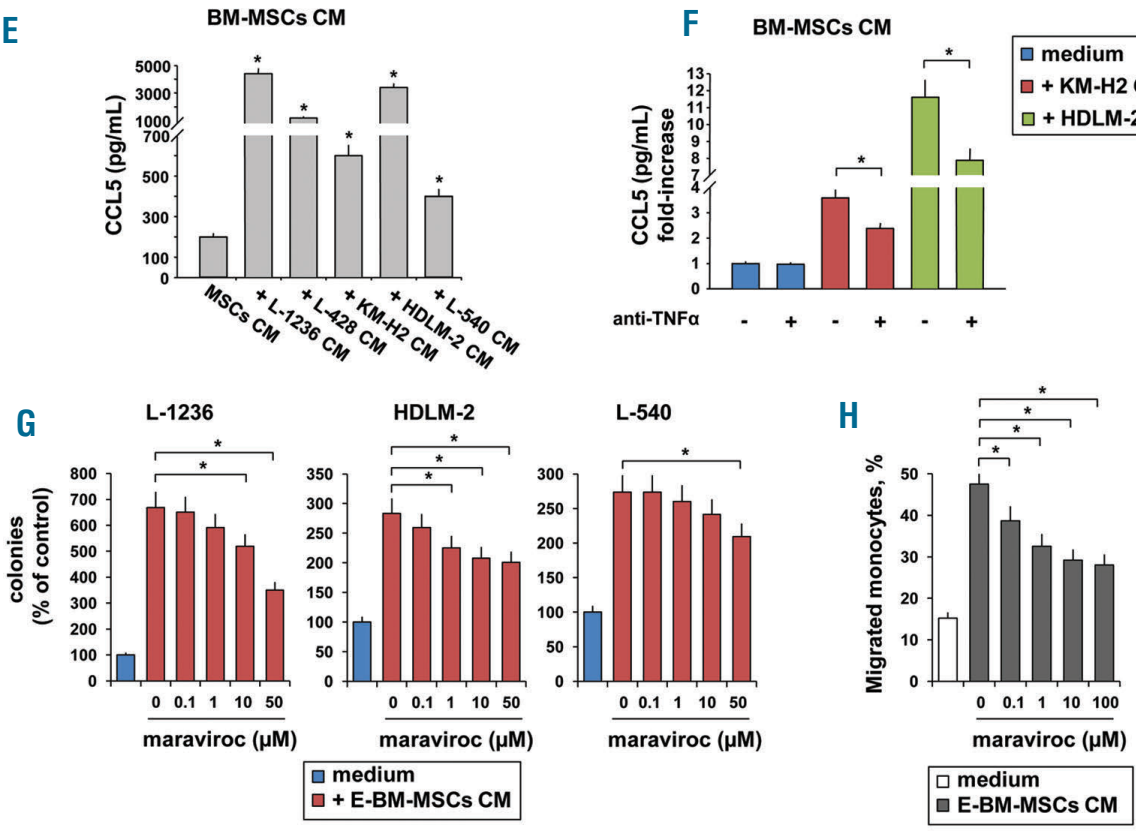

Figure 1. Maraviroc inhibits crosstalk between cHL cells and both MSCs and monocytes. (A) Percentages of BM-MSCs and AT-MSCs that migrated (in 5 h) through a fibronectin-coated Boyden chamber towards conditioned medium (CM) from L-1236 or KM-H2 cells, in the presence of increasing concentrations of maraviroc (MSCs were treated for $1 \mathrm{~h}$ prior to migration). (B) Effect of a neutralizing anti-CCL5 antibody ( $5 \mu \mathrm{g} / \mathrm{mL})$ in cHL-conditioned medium on MSC migration. Transmigrated cells were revealed using a computer-interfaced GeniusPlus microplate reader (Tecan). Results are the mean and SD of three replicate wells from three independent experiments. (C) BM-MSCs, AT-MSCs and HL-MSCs (100 cells/well; 24-well plates) were cultured in RPMI-1640 medium containing $10 \%$ cHL CM. After 9 days, cells were fixed with methanol and stained with crystal violet. (D) BM-MSCs (500 cells/well; 96-well plates) were cultured in RPMI-1640 medium containing $20 \%$ cHL CM, with or without a neutralizing anti-FGF2 $(1 \mu \mathrm{g} / \mathrm{ml})$, anti-TGF $\beta 1(2 \mu \mathrm{g} / \mathrm{mL})$ or anti-TNF $\alpha(0.5 \mu \mathrm{g} / \mathrm{mL})$ antibody. After 9 days, growth was evaluated using the MTT assay. Results are the mean and SD of three replicate wells from three independent experiments. (E) BM-MSCs were cultured for 6 days with $20 \%$ CM from L-1236, L-428, $\mathrm{KM}-\mathrm{H} 2$, HDLM-2, and L-540 cells. Then, the medium was changed with fresh medium, and 3 days later MSC CM was recovered and quantified for CCL5 by ELISA. All samples were tested in triplicate; conditioned media from three different experiments were evaluated. (F) BM-MSCs were cultured for 6 days with $20 \%$ CM (from KM$\mathrm{H} 2$ or HDLM-2 cells) in the presence or absence of a neutralizing anti-TNF $\alpha$ antibody $(0.5 \mu \mathrm{g} / \mathrm{mL})$. Then, the medium was changed and after 3 days CCL5 was quantified by ELISA. All samples were run in triplicate; conditioned media from three different experiments were evaluated. (G) Clonogenic growth. L-1236 (10 $\left.{ }^{3} / \mathrm{mL}\right)$, HDLM$2\left(5 \times 10^{2} / \mathrm{mL}\right), \mathrm{L}-540\left(2.5 \times 10^{2} / \mathrm{mL}\right)$ cells were cultured in methylcellulose-containing medium in the absence or presence of $5 \% \mathrm{E}$-BM-MSC CM and with increasing concentrations of maraviroc. After 14 days of incubation, plates were observed under phase contrast microscopy and aggregates with 40 cells or more were scored as colonies (8 replicate wells). Each experiment was done in triplicate; conditioned media from three different experiments were evaluated. (H) Percentage of CD14 monocytes that migrated (in $1 \mathrm{~h}$ ) through fibronectin-coated Boyden chambers towards medium (RPMI-1640 plus $10 \%$ FCS) or E-BM-MSC CM. Prior to migration towards E-BM-MSC CM, monocytes were pretreated with maraviroc (0.1-100 $\mu \mathrm{M})$ for $1 \mathrm{~h}$. Results are means and SD of transmigrated monocytes for three different experiments. AT-MSCs: Adipose Tissue; BM-MSCs: Bone Marrow; cHL: classical Hodgkin lymphoma; CM: conditioned medium; HL-MSCs: Hodgkin lymphoma; MSC: Mesenchymal stromal cells; E-BM-MSCs: tumor Educated. 


\section{Results}

Maraviroc inhibits crosstalk between cHL cells and both MSCs and monocytes

Conditioned medium from L-1236 and KM-H2 cHL-derived cell lines stimulated the migration of BM-MSCs (Figure 1A, left) and AT-MSCs (Figure 1A, right). Since MSCs express CCR5 (Online Supplementary Figure $S 1 A)^{35}$ and cHL cells secret CCL $5{ }^{7}$ we investigated whether this chemokine is directly involved in MSC migration. Addition of the CCR5 antagonist maraviroc (Figure 1A) or a neutralizing anti-CCL5 antibody (Figure 1B) significantly reduced the MSC migration induced by $\mathrm{cHL}$-conditioned medi um. Growth of MSCs from different sources (bone marrow, adi- pose tissue, and cHL lymph nodes) increased in the presence of cHL-conditioned medium in a dose-dependent manner (Figure 1C and Online Supplementary Figure $S 1 B$ ). This effect was partially mediated by FGF2, TGF $\beta 1$ and TNF $\alpha$ secreted by cHL cells, since addition of antibodies against these growth factors significantly, but incompletely, reduced growth (Figure 1D). cHL-conditioned medium almost totally abolished MSC senescence induced by serum starvation (Online Supplementary Figure S1C) and reduced apoptosis induced by doxorubicin treatment (Online Supplementary Figure $S 1 D$ ). To survive and proliferate, cancer cells not only recruit but also shape or "educate" normal cells. ${ }^{11}$ BM-MSCs released very low amounts of CCL5 under normal culture conditions (Figure 1E). After being cultured with cHL-conditioned medium, thereby
A

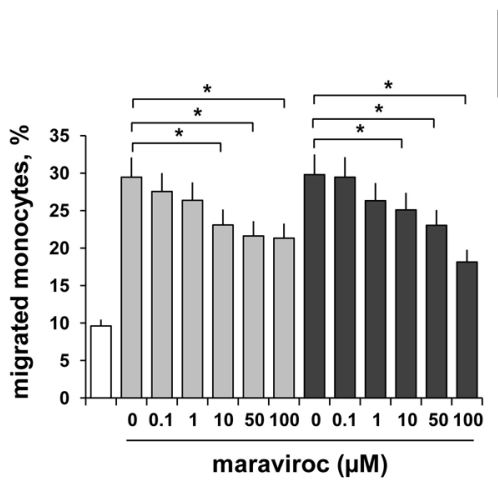

C

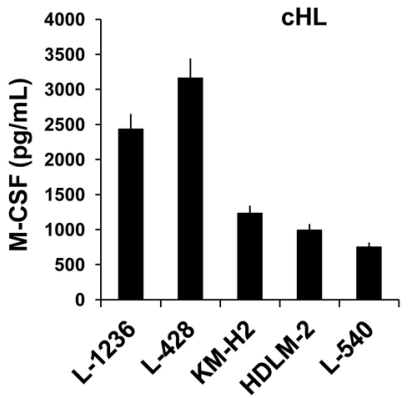

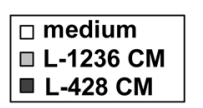

B
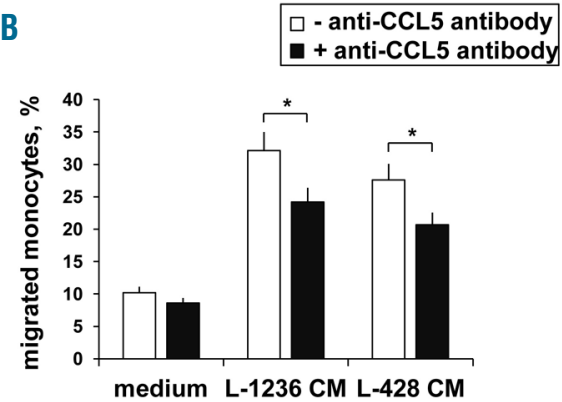

D

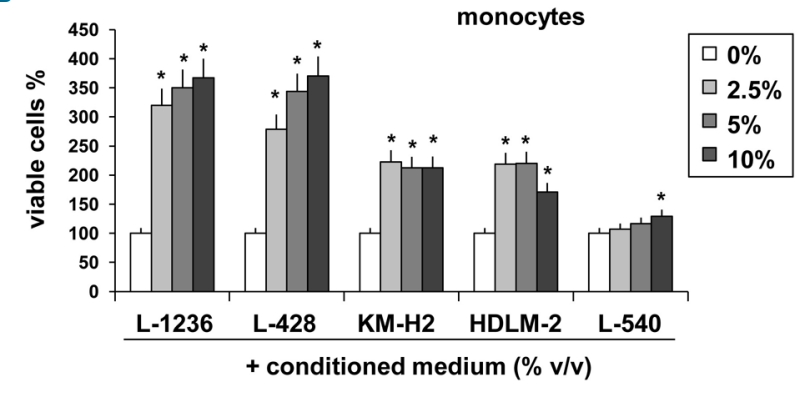

E
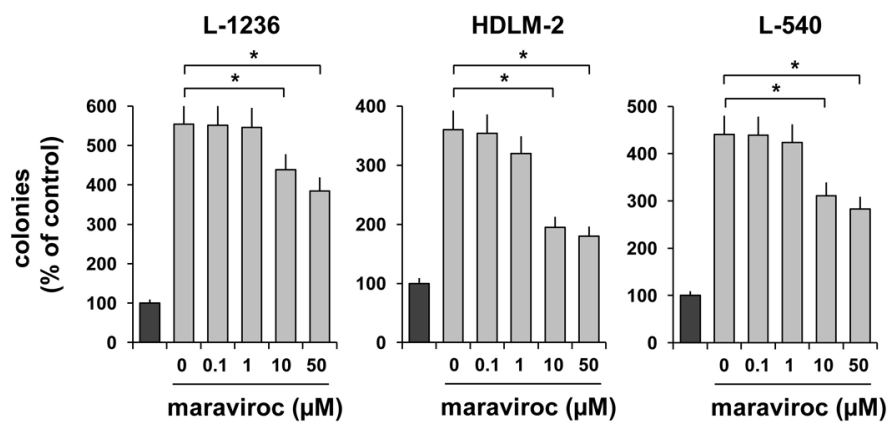

$\square$ medium

$\square+$ E-monocytes CM

Figure 2. cHL cells induce monocyte migration and proliferation. (A) Percentages of monocytes that migrated (in $1 \mathrm{~h}$ ) through fibronectin-coated Boyden chambers towards conditioned medium (CM) from L-1236 and L-428 cells, in the presence of increasing concentrations of maraviroc (monocytes were treated for $1 \mathrm{~h}$ prior to migration). (B) Effect of a neutralizing anti-CCL5 antibody $(5 \mu \mathrm{g} / \mathrm{mL})$ in cHL-conditioned medium on monocyte migration. Results are means and SD of three replicate wells from three independent experiments. (C) cHL cells $\left(2 \times 10^{5} \mathrm{cells} / \mathrm{mL}\right)$ were cultured for 3 days before CM was collected and tested for M-CSF by ELISA. All samples were run in triplicate; conditioned media from three different experiments were evaluated. (D) Monocytes (2.0x10 cells/well; 96-well flat-bottomed plates) were exposed to increasing concentrations (percentage, $\mathrm{v} / \mathrm{v}$ ) of $\mathrm{cHL} \mathrm{CM}$. After 9 days, monocyte growth was evaluated using the MTT assay. Results are mean and SD of three experiments. (E) Clonogenic growth in methylcellulose-containing medium. L-1236 $\left(10^{3} / \mathrm{mL}\right)$, HDLM-2 $\left(5 \times 10^{2} / \mathrm{mL}\right)$, L-540 $\left(2.5 \times 10^{2} / \mathrm{mL}\right)$ cells were cultured in the absence or presence of $5 \%(\mathrm{v} / \mathrm{v})$ E-monocyte $\mathrm{CM}$, with increasing concentrations of maraviroc. After 14 days of incubation, plates were observed under phase contrast microscopy and aggregates with 40 cells or more were scored as colonies (8 replicate wells). Each experiment was done in triplicate; conditioned media from three different experiments were evaluated. cHL: classical Hodgkin lymphoma; CM: conditioned medium, (E)-monocytes: tumor Educated. 
Table 1. Combination index (CI) values for L-1236 and HDLM-2 cell lines treated with maraviroc (first column) and with doxorubicin, brentuximab vedotin, cisplatin, gemcitabine or vinorelbine for $72 \mathrm{~h}$.

\begin{tabular}{|c|c|c|c|c|c|c|c|c|c|c|c|}
\hline & $\begin{array}{l}\text { MVC } \\
(\mu \mathrm{M})\end{array}$ & $\begin{array}{l}\text { Doxo } \\
\text { (ng/mL) }\end{array}$ & CI & $\begin{array}{c}\text { BV } \\
(\mu g / m L)\end{array}$ & CI & $\begin{array}{l}\text { CDDP } \\
(\mu \mathrm{M})\end{array}$ & CI & $\begin{array}{l}\text { GCB } \\
(n M)\end{array}$ & CI & $\begin{array}{c}\text { VRB } \\
\text { (ng/mL) }\end{array}$ & CI \\
\hline \multirow[t]{3}{*}{ L-1236 } & 25 & 6.0 & 0.397 & 3.8 & 0.253 & 0.13 & 0.606 & 0.15 & 0.662 & 0.13 & 0.849 \\
\hline & 50 & 12.5 & 0.594 & 7.5 & 0.357 & 0.25 & 0.955 & 0.30 & 0.912 & 0.25 & 0.946 \\
\hline & 100 & $* 25.0$ & 0.628 & *15.0 & 0.495 & $* 0.50$ & 1.152 & $* 0.60$ & 1.302 & $* 0.50$ & 1.051 \\
\hline \multirow[t]{3}{*}{ HDLM-2 } & 25 & 3.1 & 0.512 & 75 & 0.373 & 0.31 & 0.571 & 0.80 & 0.699 & 0.25 & 0.818 \\
\hline & 50 & 6.2 & 0.526 & 150 & 0.478 & 0.63 & 0.706 & 1.60 & 1.054 & 0.50 & 0.887 \\
\hline & 100 & *12.5 & 0.753 & *300 & 0.655 & $* 1.25$ & 1.169 & $* 3.20$ & 1.173 & $* 1.00$ & 1.045 \\
\hline
\end{tabular}

Cell viability was determined by trypan blue dye exclusion. Combination indexes were calculated using CalcuSyn software. *Indicates the IC50 for each drug. (IC50) Concentration required for $50 \%$ in vitro inhibition of growth. MVC: maraviroc; Doxo: doxorubicin; BV: brentuximab vedotin; CDDP: cisplatin; GCB: gemcitabine;VRB: vinorelbine

becoming tumor educated (E-BM-MSCs), they strongly secreted CCL5 (Figure 1E). This response was partially reduced by treatment with an anti-TNFa antibody (Figure 1F). Education of BMMSCs did not, however, induce the secretion of CCL3 or CCL4 (data not shown).

Conditioned medium from E-BM-MSCs increased the clonogenic growth of L-1236, HDLM-2 and L-540 cells, and this effect was reduced by maraviroc in a dose-dependent manner (Figure 1G and Online Supplementary Figure S2A). This effect was due to CCL5-CCR5 interactions, because it was partially inhibited by inclusion of a neutralizing anti-CCL5 antibody (Online Supplementary Figure S2B). Conditioned medium from BM-MSCs educated with L-1236-conditioned medium (and thereby containing CCL5; Figure 1E) increased the migration of CD14+ monocytes, and this effect was reduced in a dose-dependent manner by maraviroc (Figure $1 \mathrm{H}$ ).

Taken together, these results suggest that E-BM-MSCs, by secreting CCL5, stimulate tumor growth as well as monocyte recruitment in the TME.

\section{cHL cells induce monocyte migration and proliferation}

cHL-conditioned medium increased CCR5 expression in monocytes (Online Supplementary Figure S3A) and enhanced their migration through fibronectin-coated Boyden chambers (Online Supplementary Figure S3B). Representative photomicrographs of transmigrated monocytes (red Fast-Dil colored cells) are shown in Online Supplementary Figure S3C. This enhanced monocyte migration was significantly reduced when maraviroc (Figure 2A) or a neutralizing anti-CCL5 antibody (Figure 2B) was added. cHL cells, especially L-1236 and L-428 cells, secreted macrophage colonystimulating factor (M-CSF) (Figure 2C), a cytokine involved in monocyte proliferation and differentiation. In accordance, conditioned medium from cHL cells increased monocyte growth (Figure 2D and Online Supplementary Figure S3D). E-monocytes secreted CCL3 and low amounts of CCL4 and CCL5 (Online Supplementary Figure S3E). Treatment of L-1236, HDLM-2 and L-540 cells with conditioned medium from E-monocytes increased the number of viable tumor cells (Online Supplementary Figure S3F) and stimulated clonogenic growth (Figure $2 \mathrm{E}$ ), but this growth was inhibited by maraviroc treatment (Figure 2E and Online Supplementary Figure S3G).

\section{Reprogramming of monocytes by $\mathrm{cHL}$ cells}

TAMs express high levels of CD206, PD-L1 and IDO; they secrete IL-10, CCL17/TARC and TGF- $\beta$, and can inhibit growth of activated $\mathrm{T}$ cells. ${ }^{16}$ When monocytes were cultivated in conditioned medium from L-1236 or L-428 cells, they upregulated the secretion of IL-10, CCL17, and TGF $\beta$ (Figure 3A) and increased the expression of CD206, PD-L1, and IDO (Figure 3B). Conditioned medium from L-540 cells did not induce IL-10 or CCL17 secretion or IDO expression by monocytes, but did enhance CD206, PD-L1, and especially TGF $\beta$ secretion (Figures $3 \mathrm{~A}-\mathrm{B}$ ). Conditioned medium from E-monocytes slowed, in a dose-dependent manner, the growth of phytohemagglutinin-activated lymphocytes (Figure $3 \mathrm{C}$ ). These results demonstrate that $\mathrm{cHL}$ cells recruit, induce proliferation, and then reprogram monocytes towards an immunosuppressive phenotype.

\section{Maraviroc slows tumor cell growth and synergizes with doxorubicin and brentuximab vedotin}

Considering that $\mathrm{CHL}$ cells express a functional CCR5 receptor, we evaluated cHL cell growth in the presence of increasing concentrations of maraviroc. This treatment slightly slowed the growth of cHL cells (Figure 4A) and slightly increased the percentage of cells in the G1 phase of the cell cycle (Figure 4B and Online Supplementary Figure S4A). On the contrary, maraviroc treatment did not induce apoptosis-necrosis as shown by the lack of change in annexin-V and 7-AAD staining (Online Supplementary Figure $S 4 B$ ) and in levels of activated caspase-3/7 (Online Supplementary Figure $54 \mathrm{C}$ ).

Maraviroc $(25,50,100 \mu \mathrm{M})$ synergized with doxorubicin and brentuximab vedotin as indicated by the combination index as being $<0.9$ in all three conditions tested (Table 1 and Online Supplementary Figure S5A-B). Moreover, maraviroc exerted additive or antagonist effects (combination index $\geq 0.9$ ) in combination with cisplatin, gemcitabine and vinorelbine (Table 1). Synergistic effects with both doxorubicin and brentuximab vedotin were also obtained using ten-fold lower maraviroc concentrations $(2.5,5$, $10 \mu \mathrm{M})$, but not with hundred-fold lower concentrations $(0.25$, 0.5, $1.0 \mu \mathrm{M})$ (Online Supplementary Table S2).

\section{Maraviroc inhibits 3D heterospheroid formation}

To mimic TME interactions, we co-cultured cHL cells with MSCs and monocytes in a non-adherent, 3D setting in polyHEMA-coated wells. The cells were dispersed in the medium at time 0 but at $24 \mathrm{~h}$ had started to self-assemble into 3D heterospheroids (Figure 4C and Online Supplementary Figure S6A). Heterospheroids containing all three cell types secreted high levels of CCL5, and those containing only cHL cells (either L-1236 or HDLM-2) and HL-MSCs also had high CCL5 secretion, whereas the combinations of just monocytes with cHL cells or with HLMSCs expressed low levels (Figure 4D). However, when combined with HL-MSCs and cHL cells, monocytes were able to induce a further increase of CCL5 secretion (Figure 4D). Next, we evaluated the effects of maraviroc on the self-assembling ability and viability of heterospheroids. Maraviroc inhibited the self- 
A

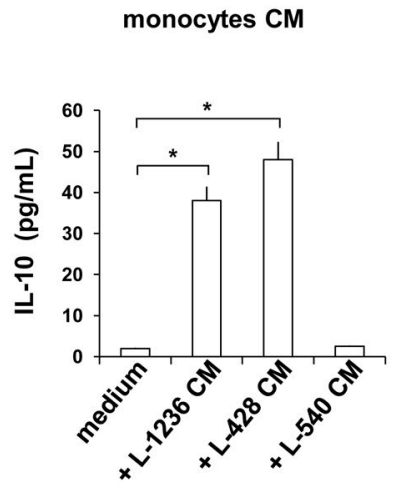

B

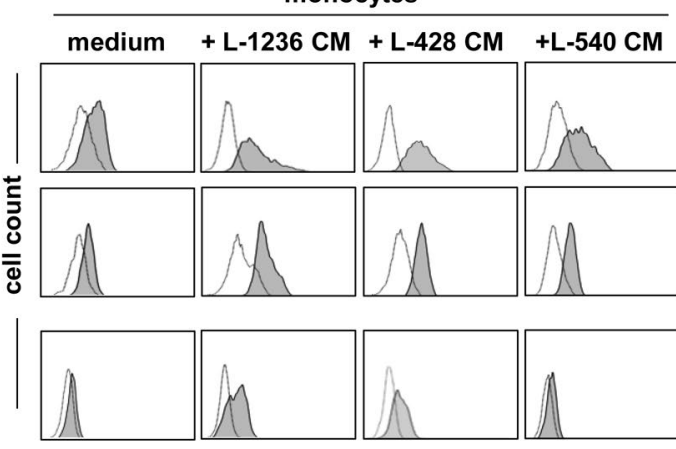

fluorescence intensity
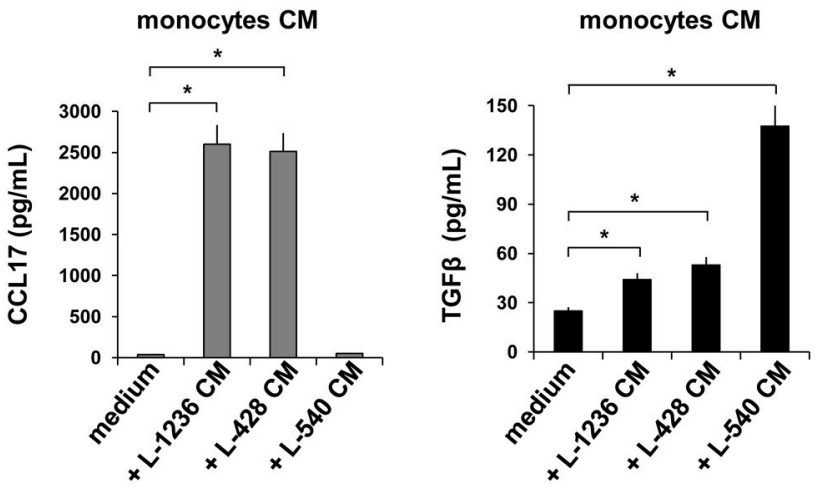

C CD206

PD-L1 IDO

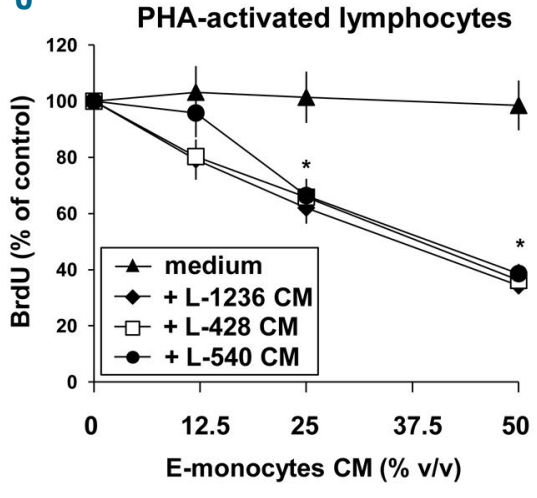

Figure 3. Monocyte conversion towards immunosuppressive E-monocytes by cHL cells. (A) Purified CD14 monocytes were cultured for 6 days in the absence or presence of $20 \%$ conditioned medium (CM) from L-1236, L-428, and L-540 cells (to convert monocytes into E-monocytes), then washed and cultured for another 72 $\mathrm{h}$ in fresh medium. This E-monocyte CM was recovered for IL-10, CCL17, and TGF $\beta$ ELISAs. Results are means and SD of three independent experiments. (B) Representative flow cytometric histograms showing surface expression of CD206 and PD-L1 and intracellular expression of IDO in monocytes (control) and E-monocytes (treated with $\mathrm{CHL} \mathrm{CM).} \mathrm{(C)} \mathrm{E-monocyte} \mathrm{CM}$ was tested for immunosuppressive activity. Phytohemagglutinin (PHA)-activated lymphocytes were treated with increasing concentrations (percentage, v/v) of monocyte CM and E-monocyte CM. After $72 \mathrm{~h}$, growth was assayed using the Cell Proliferation ELISA, BrdU. All samples were tested in triplicate; conditioned media from three different experiments were evaluated. cHL: classical Hodgkin lymphoma; CM: conditioned medium, (E)-monocytes: tumor Educated.

assembling of cHL, HL-MSCs and monocytes into heterospheroids (Figure 4E). It also reduced the total number of viable cells in the heterospheroids (Figure 4F). Considering this reduced viability, and the finding that maraviroc synergized with doxorubicin (Table 1), we applied drug combinations to the heterospheroids and found that doxorubicin and maraviroc exerted synergistic activity (combination index $<1$ ) against heterospheroids too (Online Supplementary Figure S6B). When cHL cells (CD30+) were recovered from heterospheroids by trypsinization and purification with anti-CD30 beads, fewer viable tumor cells were recovered from maraviroc-treated than from untreated heterospheroids (Online Supplementary Figure S6C). The cells from treated heterospheroids produced fewer colonies (Online Supplementary Figure $S 6 D)$ and were proportionately more in G1 than in G2M phase than untreated cells (Online Supplementary Figure S6E).

\section{Maraviroc slows the growth of $\mathrm{cHL}$ tumor xenografts and reduces infiltrated TAMs}

To analyze the anticancer activity of maraviroc in vivo, we studied L-540 tumor cell xenografts in female athymic nude mice, treated every day with an intraperitoneal injection of $10 \mathrm{mg} / \mathrm{kg}$ maraviroc or vehicle. By day 12 , untreated tumors had grown to a mean volume of $880 \mathrm{~mm}^{3}\left(\mathrm{SD}=88 \mathrm{~mm}^{3}\right)$, whereas maraviroctreated tumors were more than $50 \%$ smaller $\left(435 \pm 75 \mathrm{~mm}^{3}\right.$; $P<0.0001$, Student's $t$-test; Figure 5A and Online Supplementary
Figure S7A). Maraviroc treatment was not toxic as the animals were normal on physical inspection and had similar weight to untreated animals (Figure 5B). Maraviroc-treated mice had significantly better "survival" (i.e., tumor volume $<800 \mathrm{~mm}^{3}$ ) than untreated mice ( $P=0.002, \log$ rank test) (Online Supplementary Figure $S 7 B$ ). Since maraviroc inhibited the migration of monocytes in vitro, we evaluated whether similar activity was also detectable in vivo by examining infiltrating TAMs $\left(\mathrm{CD}^{+}\right)$in L-540 tumor xenografts. Immunofluorescence analysis of CD30 on tissue sections showed no difference between untreated and maraviroctreated mice (Online Supplementary Figure S7C). However, the animals differed substantially in staining for CD68, a marker of TAM infiltration, which was almost completely absent in maraviroctreated mice (Figure 5C-D and Online Supplementary Figure S7D).

Similar results were obtained when male NSG mice were injected with L-428 tumor cells (Figure 5E-H). In particular, maraviroc treatment reduced xenograft growth by about $60 \%$ (Figure $5 \mathrm{E}$ ), without weight loss (Figure 5F), and it reduced CD68 staining by 75\% (Figure 5G-H and Online Supplementary Figure S7D).

\section{High CCL5 expression positively correlates with CD68} and poor survival

To confirm our in vitro and in vivo results, we studied cHL tissues from 65 patients (Online Supplementary Table S1). cHL tissues had a median CCL5 expression level of $12 \%$ positive pixels (range, $0 \%$ - 
$44 \%$ ) and a median CD68 expression level of $17 \%$ positive pixels (range, 0\%-58\%) (Figure 6A). No difference in CCL5 expression was found in EBV-positive versus EBV-negative HL samples (data not shown). On the contrary, a significant correlation between CCL5 and CD68 levels was found (Spearman $\mathrm{r}=0.251, P=0.0487$ ) (Figure $6 \mathrm{~B})$. Patients were then divided into three arbitrary categories of CCL5 expression: low (0\%-10\%), medium (11\%-20\%) and high
(21\%-100\%). These groups had 24, 22, and 16 patients, respectively (data missing for 3 patients). A Kaplan-Meier plot showed that the group of patients with high CCL5 expression had significantly worse survival $(P=0.0072)$ than patients with low or medium expression (Figure 6C). The hazard ratio for progression-free survival of low/medium CCL5 expression to that of high expression was 0.015 ( $P=0.0016$; 95\% CI, 0.0011-0.2064).
A

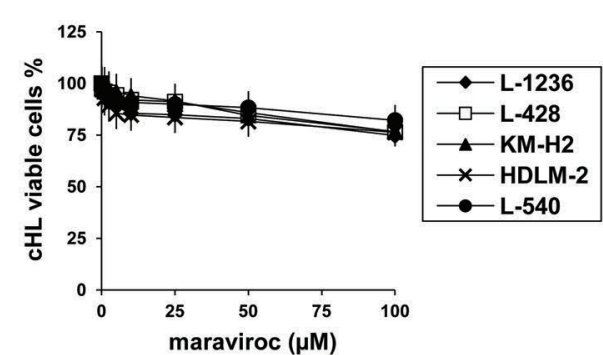

C

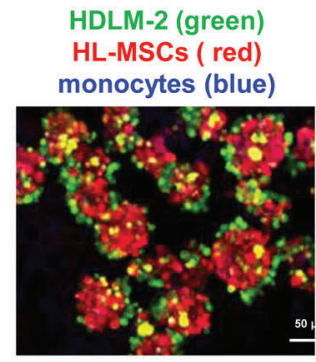

3D heterospheroids
B

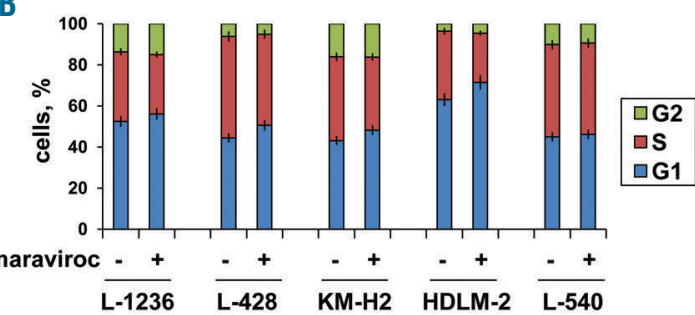

D
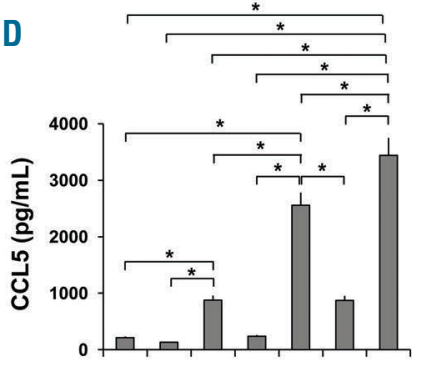

$\mathrm{L}-1236+-\quad++-+$

HL-MSCs - - + - + + +

monocytes - + - + - + +

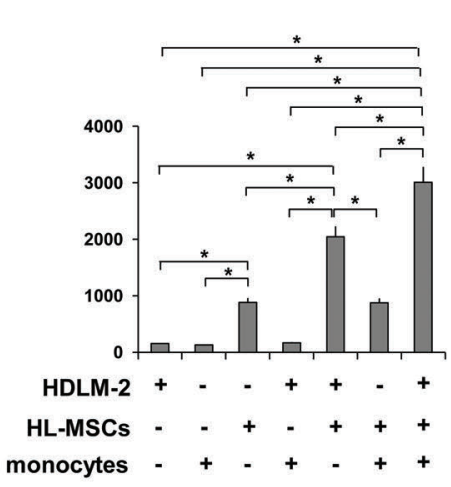

$E$

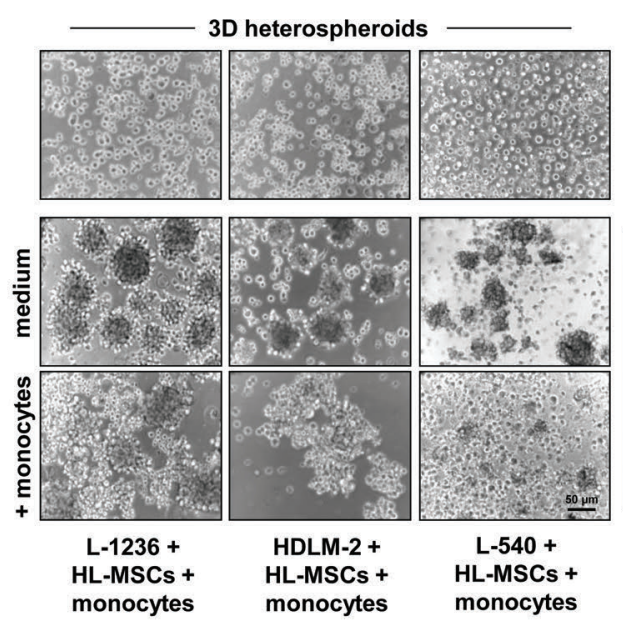

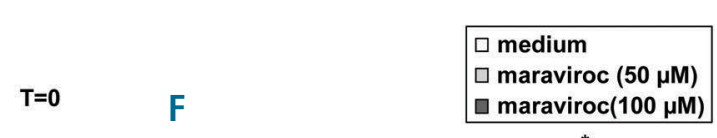

$T=24 h$

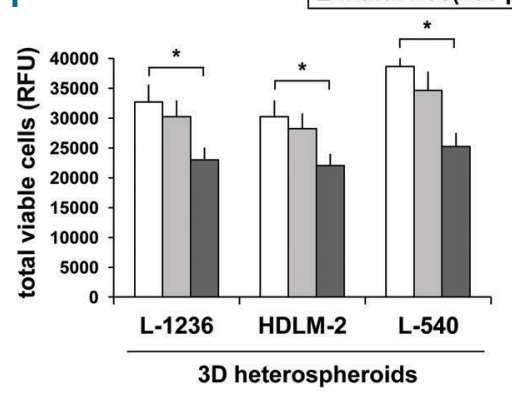

Figure 4. Maraviroc slows tumor cell growth and inhibits heterospheroid formation. (A) $\mathrm{CHL}$ cells $\left(2 \times 10^{5}\right.$ cells $\left./ \mathrm{ml}\right)$ were cultured with increasing concentrations of maraviroc for $72 \mathrm{~h}$, and viable cells were counted by trypan blue dye exclusion. Results are means and SD of three replicate wells from three independent experiments. (B) Percentages of $\mathrm{cHL}$ cells in the various cell cycle phases after a $24 \mathrm{~h}$ treatment with maraviroc (100 $\mu \mathrm{M})$. Results are means and SD of at least three experiments. (C) Representative image of 3D heterospheroids generated by plating HDLM-2 cells (stained green with CFSE), MSCs (red with fluorescent Dil), and monocytes (blue with DiD) under non-adherent conditions (poly-HEMA-coated wells). (D) L-1236 or HDLM-2 cells, HL-MSCs and monocytes were cultured in RPMI 1640 medium plus $1 \%$ FCS, alone or in combination under non-adherent conditions. After 4 days, conditioned medium was collected for CCL5 ELISAs; three different experiments were evaluated. (E) Heterospheroids generated by co-cultivation of cHL cells (L-1236, HDLM-2, or L-540 cells) with HL-MSCs and monocytes under nonadherent conditions in the presence or absence of maraviroc $(100 \mu \mathrm{M})$ and photographed after $24 \mathrm{~h}$ using an inverted microscope (Eclipse TS/100, Nikon). (F) Heterospheroids (cHL + HL-MSCs + monocytes) were cultured with and without maraviroc. After $48 \mathrm{~h}$, viable cells were evaluated using PrestoBlue Cell Viability Reagent. Relative fluorescence units (RFU). Values are means and SD of three experiments. cHL: classical Hodgkin lymphoma; (HL)-MSCs: Hodgkin lymphoma; MSC: mesenchymal stromal cells; 3D: three dimensional. 


\section{Discussion}

The TME plays an active role in $\mathrm{CHL},{ }^{11}$ suggesting the possibility of developing alternative treatment strategies that target not only tumor cells, but also the TME's protective effects. ${ }^{11,22}$ Here, we found that maraviroc, a CCR 5 antagonist, inhibited cHL cell recruitment of monocytes and MSCs, reduced the cHL cell growth-promoting effects of CCR5 ligands secreted by monocytes and MSCs, synergized with doxorubicin and brentuximab vedotin, and decreased cHL tumor xenograft growth and monocyte infiltration in vivo. In CHL patients, high CCL5 levels corre- lated with monocyte infiltration and poor prognosis.

Our in vitro results suggest that there is a "domino effect" within the CHL TME: tumor cells, by secreting CCL5, among other molecules, recruit, expand, and educate MSCs and monocytes; these cells, in turn, secrete CCR5 ligands (i.e., CCL3 and CCL4) to recruit other normal cells and stimulate the growth of tumor cells, which reprogram (educate) monocytes to become immunosuppressive TAMs. A schematic view of the possible mechanisms leading to $\mathrm{cHL}$ cell proliferation and TME formation by CCR 5 ligands, and the counteracting effects of maraviroc, is shown in Figure 7.
A

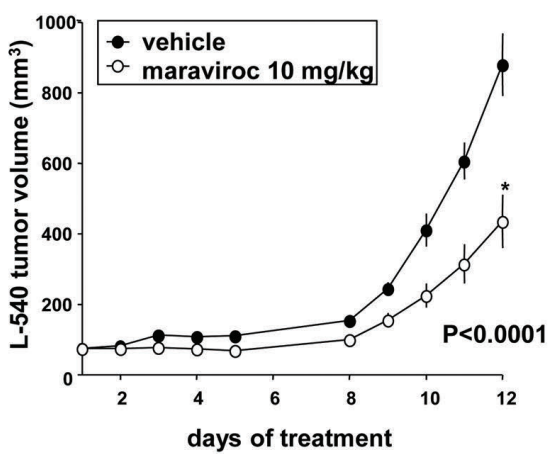

C

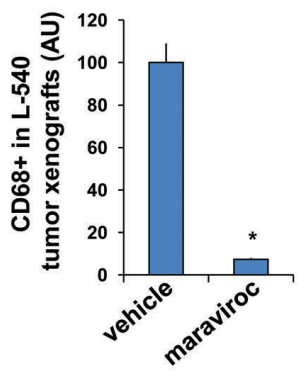

E

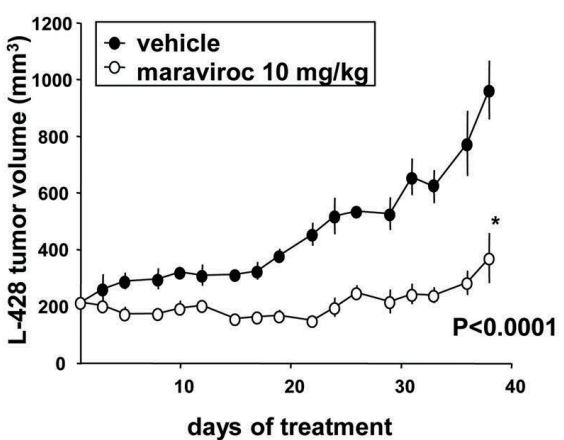

G

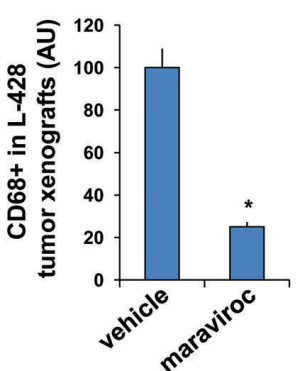

B
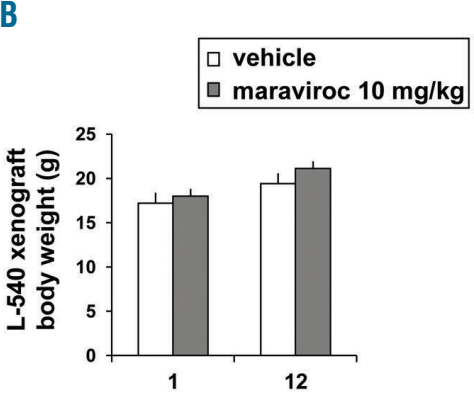

day of treatment

D

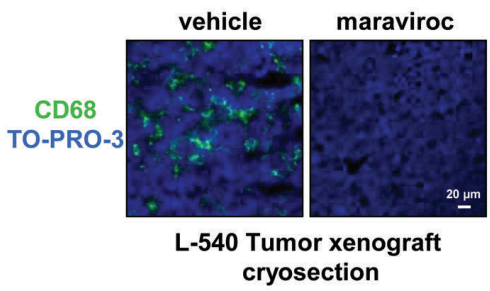

$\mathbf{F}$
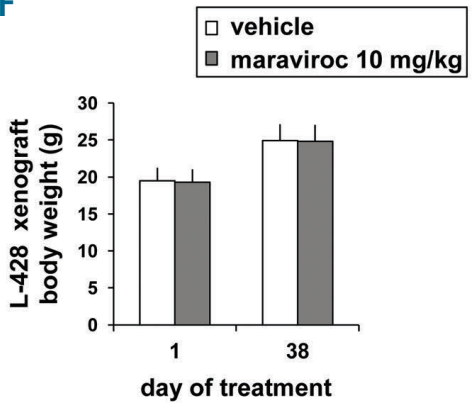

H

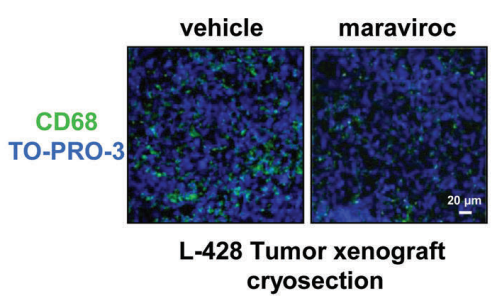

Figure 5. Maraviroc reduces $\mathrm{cHL}$ xenograft growth and TAM infiltration. (A-D) Xenografts in nude mice inoculated with $\mathrm{L}$ 540 cells $\left(20 \times 10^{6}\right.$ cells/animal) and treated every day with an intraperitoneal injection of $10 \mathrm{mg} / \mathrm{kg}$ maraviroc $(n=5)$ or vehicle $(n=5)$. (A) Xenograft tumor growth. (B) Body weights of xenografted mice. (C) Quantification of $\mathrm{CD}^{+} 8^{+}$staining in immunofluorescent cryosections using Volocity software provided by PerkinElmer (arbitrary units). Data are means and SD. (D) Immunofluorescent photomicrographs of $\mathrm{CD}^{+} 8^{+}$staining in tumor cryosections from maraviroc-treated and untreated xenografted mice. Nuclei were stained with TO-PRO-3 dye. Representative images were acquired using a confocal microscope (Leica DM IRE2). (E-H) Xenografts in NSG mice inoculated with $\mathrm{L}-428$ cells $\left(10 \times 10^{6}\right.$ cells/animal) and treated every other day with an intraperitoneal injection of $10 \mathrm{mg} / \mathrm{kg}$ maraviroc $(n=5)$ or vehicle $(n=5)$. (E) Xenograft tumor growth. (F) Body weights of xenografted mice. (G) Quantification of $\mathrm{CD}^{+} 8^{+}$staining in immunofluorescent cryosections using Volocity software (arbitrary units). Data are means and SD. (H) Immunofluorescent photomicrographs of $\mathrm{CD}^{+} 8^{+}$staining in tumor cryosections from maraviroc-treated and untreated xenografted mice. Nuclei were stained with TO-PRO-3 dye. Representative images were acquired using a confocal microscope (Leica DM IRE2). NSG: NOD/SCID gamma chain deficient; TAM: tumor associated macrophages. 
We found that conditioned medium from cHL cells increased the growth of MSCs from different sources, and maraviroc decreased MSC recruitment by cHL cells. Thus, the development of fibrosis in cHL tissues could be explained by recruitment of MSCs by cytokines, including CCL5, secreted by primary tumors and expansion or activation by FGF2, TGF $\beta$ or TNF $\alpha$ secreted by tumor cells. Moreover, our findings that $\mathrm{cHL}$ education of MSCs (E-MSCs) increased the secretion of CCL5 and that maraviroc reduced monocyte recruitment by E-MSC-conditioned medium suggest an active role of E-MSCs in TME formation. In this perspective, MSCs of the cHL TME not only may down-regulate anti-tumor immune responses through NKG2D-NKG2DL interactions, ${ }^{36,37}$ but may also enhance the number of infiltrated TAMs by secreting CCL5 and, consequently, supporting tumor progression.

Macrophages seem to be involved in the pathogenesis of cHL, since high levels of TAMs as well as the absolute monocyte count in the blood correlate with an unfavorable clinical outcome. . $^{17,20,21,38}$ Despite the importance of monocyte levels in TME, their education (i.e., conditioning by tumor cells) seems an essential prerequisite for their pro-tumor activity. ${ }^{39,40}$ Recently, it was demonstrated that conditioned medium from cHL cell lines induced an immunosuppressive phenotype in macrophages obtained by pre-cultivation of monocytes with M-CSF or GM-
A
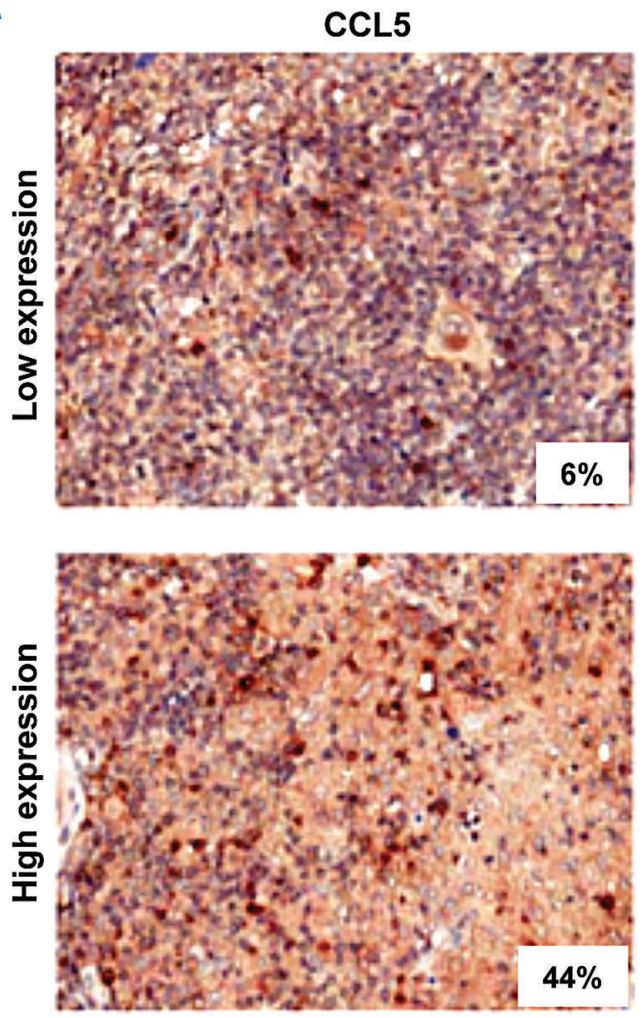

CD68
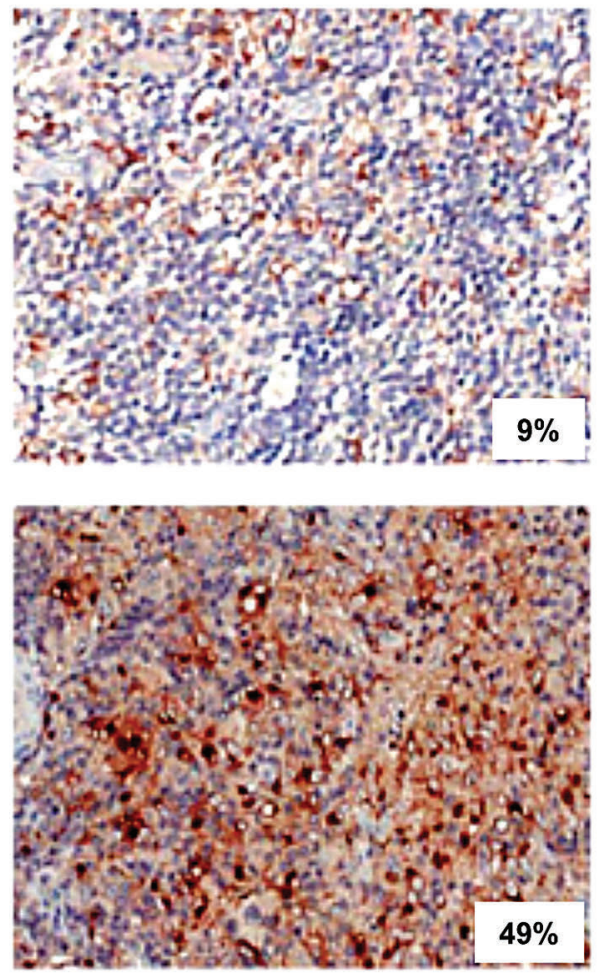

B

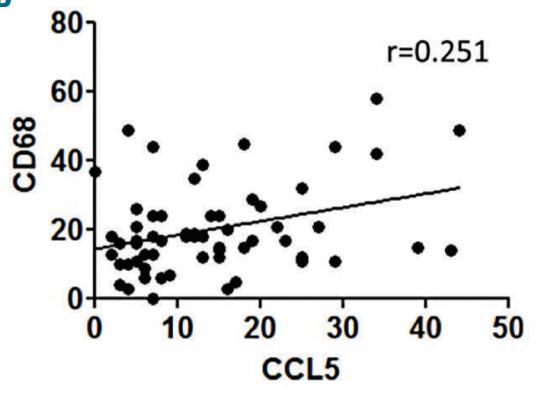

C

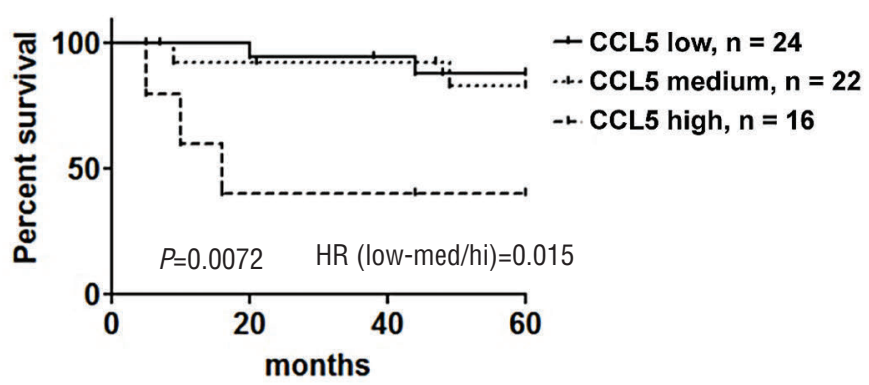

Figure 6. CCL5 expression positively correlates with CD68 expression in human $\mathrm{CHL}$ tissues and with lower progression-free survival in $\mathrm{cHL}$ patients. (A) Representative photomicrographs of CHL tissues stained for CCL5 and CD68 (marker of macrophages/monocytes). Top row, a case with low expression; bottom row, a case with high expression. (B) Correlation of expression levels of CCL5 and CD68 in $65 \mathrm{cHL}$ patients. Spearman $\mathrm{r}=0.251 ; P=0.0487$. (C) Kaplan-Meier survival plot for 5-year progression-free survival in cHL patients, subdivided according to the percentage of CCL5 positivity (low, 0\%-10\%; medium, 11\%-20\%; high, 21\%-100\%). Patients with high CCL5 expression had worse survival ( $P=0.0072$, HR=0.015 vs. low-medium levels). cHL: classical Hodgkin lymphoma; HR: hazard ratio; $r$ : Spearman correlation coefficient. 
CSF. ${ }^{16}$ Consistent with the finding that $\mathrm{cHL}$ cells secrete $\mathrm{M}$-CSF, the education of monocytes by cHL-cell conditioned medium, also without a preconditioning with $M$ CSF or GM-CSF, ${ }^{16,17}$ was sufficient to shape monocytes to secrete and express immunosuppressive molecules, including IDO and PD-L1, and to inhibit PHA-activated lymphocyte growth.

Recently, it was found that, in cHL tissues, TAMs are not randomly distributed; in fact, $\mathrm{PD}-\mathrm{L}^{+}$TAMs lie in greater proximity to $\mathrm{PD}-\mathrm{L} 1^{+}$tumor cells and $\mathrm{PD}^{+} \mathrm{T}$ cells preferentially localize in proximity to $\mathrm{PD}-\mathrm{L} 1^{+} \mathrm{TAMs}$, suggesting a model in which the inflammatory microenviron- ment of cHL is highly organized with PD-L1+ TAMs immediately surrounding Hodgkin and Reed-Sternberg cells to engage $\mathrm{PD}-1^{+} \mathrm{T}$ cells and augment immune suppression. ${ }^{41}$ Thus, our results suggest that cHL cells, by inducing PD-L1 expression in monocytes, contribute to the building of the immunosuppressive niche. ${ }^{41}$ Maraviroc, by reducing monocyte recruitment, may counteract this phenomenon.

Since current two-dimensional (2D) in vitro methods often fail to adequately replicate tumor cell interactions with the TME and to properly assess drug activity, here, to evaluate the effects of maraviroc, we developed and

\section{Tumor growth}

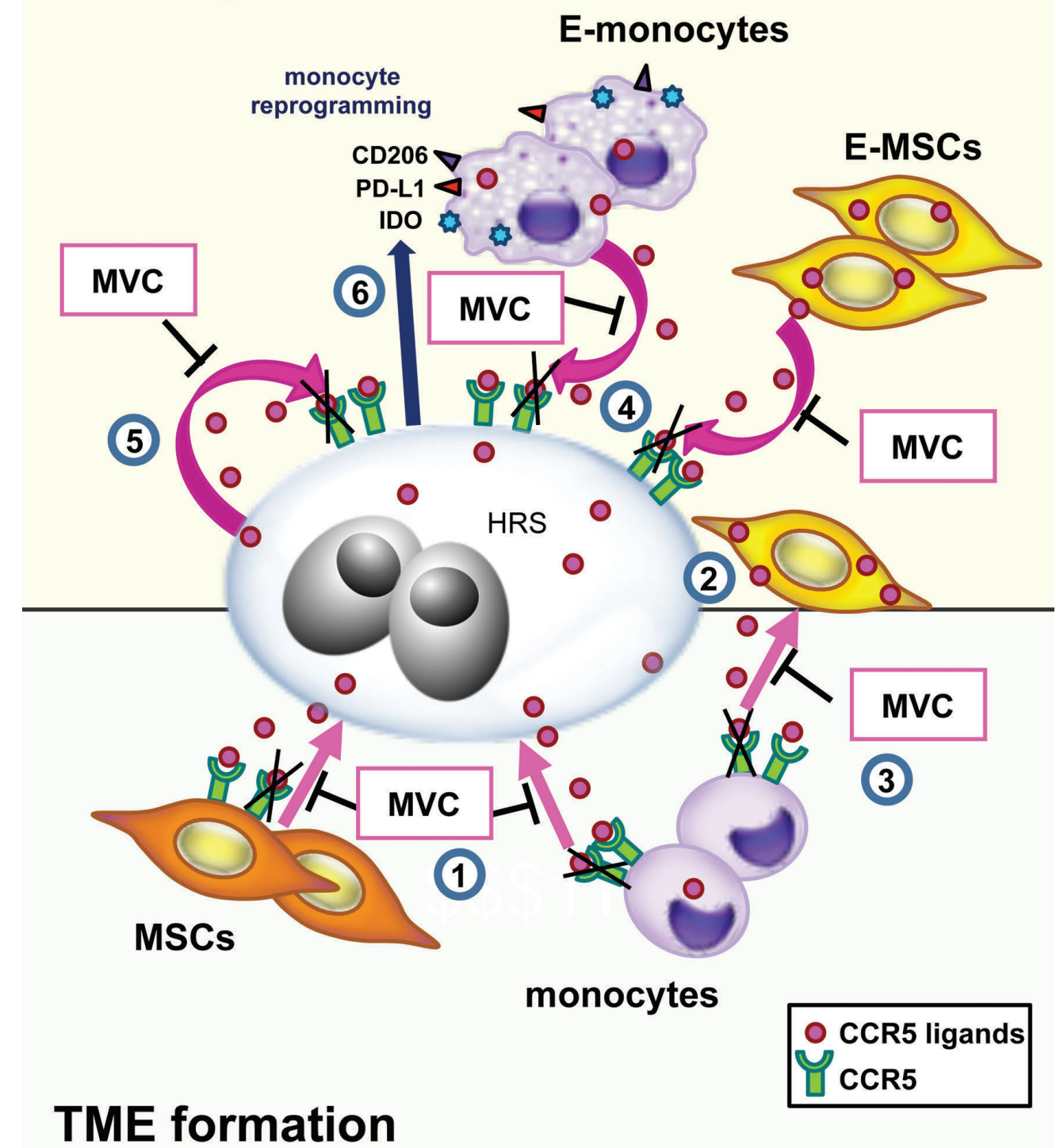

Figure 7. Proposed mechanism for the inhibitory effects of the CCR5 antagonist maraviroc on TME formation and cHL tumor growth. (1) Maraviroc, a CCR5 antag onist, inhibits the recruitment of monocytes and MSCs by cHL cells (Hodgkin and Reed/Sternberg, HRS). (2) The tumor "education" of MSCs cells (E-MSCs) induces the secretion of CCL5. (3) Maraviroc inhibits the recruitment of monocytes by E-MSCs secreting CCL5. (4) Maraviroc decreases the cHL cell growth-promoting effects of CCR5 ligands secreted by tumor educated-monocytes (E-monocytes) and E-MSCs (CCL5+). (5) Maraviroc inhibits the growth of cHL cells (autocrine growth). (6) cHL: classical Hodgkin lymphoma; HRS: Hodgkin and Reed/Sternberg; MVC: Maraviroc; MSC: Mesenchymal stromal cells; (E)-MSCs: tumor Educated; (E)-monocytes: tumor Educated; TME: tumor microenvironment. 
used a three-dimensional (3D) multicellular heterospheroid model ${ }^{33}$ formed by tumor cells, monocytes and MSCs. By using 3D heterospheroids, in which tumor cells and different types of normal cells interact and organize their positions, we found that interactions between MSCs, monocytes and cHL cells increased the overall secretion of CCL5. Maraviroc decreased heterospheroid selfassembling, cell viability and cHL clonogenic growth ability, suggesting that it may counteract TME formation and, as a consequence, its protective effects. In a mouse xenograft model, maraviroc decreased the in vivo growth of both L-540 and L-428 cells by more than $50 \%$ and reduced monocyte infiltration without apparent toxicity to the animals. These findings confirm that CCR5 signaling contributes to determining the fate of $\mathrm{cHL}$ tumor cells.

Recently, Jiao et al..$^{42}$ demonstrated that maraviroc dramatically enhanced cell killing of CCR $5^{+}$breast cancer cells by the DNA-damaging chemotherapeutic agent doxorubicin. Here, maraviroc synergized not only with doxorubicin, but also with brentuximab vedotin. These results suggest that CCR 5 inhibitors, by enhancing the activity of other drugs, may allow a dose reduction of the two chemotherapeutic agents already in use in cHL patients.

As shown here, CCL5 expression levels positively correlated with CD68 levels in cHL tissues, and patients with high CCL5 levels had lower progression-free survival than patients with low or medium expression of this chemokine; this difference may be due to the higher level of macrophages recruited and then reprogrammed by $\mathrm{cHL}$ cells towards immunosuppressive tumor-associated PDL1-positive macrophages as well as by enhanced tumor growth by CCL5.

In conclusion, in light of the significant reduction of tumor mass obtained with maraviroc alone, its low toxicity and ability to inhibit monocyte infiltration, our results provide the rationale for its clinical assessment in $\mathrm{cHL}$, as a single agent or in combination therapy.

\section{Funding}

This work was supported by grant IG 15844 from the Italian Association for Cancer Research (to D.A.) and by an Intramural Grant (5X1000CRO-2011).

\section{Acknowledgments \\ Valerie Matarese provided scientific editing.}

\section{References}

1. Chow MT, Luster AD. Chemokines in cancer. Cancer Immunol.Res. 2014;2(12):11251131.

2. Aldinucci D, Colombatti A. The inflammatory chemokine CCL5 and cancer progression. Mediators Inflamm. 2014; 2014:292376.

3. Zhang Q, Qin J, Zhong L, et al. CCL5mediated Th2 immune polarization promotes metastasis in luminal breast cancer. Cancer Res. 2015;75(20):4312-4321.

4. Wang LH, Lin CY, Liu SC, et al. CCL5 promotes VEGF-C production and induces lymphangiogenesis by suppressing miR507 in human chondrosarcoma cells. Oncotarget. 2016;7(24):36896-36908.

5. Atretkhany KN, Drutskaya MS, Nedospasov SA, Grivennikov SI, Kuprash DV. Chemokines, cytokines and exosomes help tumors to shape inflammatory microenvironment. Pharmacol Ther. 2016;168:98-112.

6. Ban Y, Mai J, Li X, et al. Targeting autocrine CCL5-CCR5 axis reprograms immunosuppressive myeloid cells and reinvigorates antitumor immunity. Cancer Res. 2017; 77(11):2857-2868.

7. Aldinucci D, Lorenzon D, Cattaruzza L, et al. Expression of CCR5 receptors on ReedSternberg cells and Hodgkin lymphoma cell lines: involvement of CCL5/Rantes in tumor cell growth and microenvironmental interactions. Int J Cancer. 2008;122(4):769776.

8. Mathas S, Hartmann S, Kuppers R. Hodgkin lymphoma: pathology and biology. Semin Hematol. 2016;53(3):139-147.

9. Linke F, Harenberg M, Nietert MM, et al. Microenvironmental interactions between endothelial and lymphoma cells: a role for the canonical WNT pathway in Hodgkin lymphoma. Leukemia. 2017;31(2):361-372.

10. Celegato M, Borghese C, Casagrande N, et al. Preclinical activity of the repurposed drug Auranofin in classical Hodgkin lymphoma. Blood. 2015;126(11):1394-1397.

11. Aldinucci D, Celegato M, Casagrande N. Microenvironmental interactions in classical Hodgkin lymphoma and their role in promoting tumor growth, immune escape and drug resistance. Cancer Lett. 2016;380(1):243-252.

12. Wein F, Weniger MA, Hoing $B$, et al. Complex immune evasion strategies in classical Hodgkin lymphoma. Cancer Immunol Res. 2017;5(12):1122-1132.

13. Poggi A, Musso A, Dapino I, Zocchi MR. Mechanisms of tumor escape from immune system: Role of mesenchymal stromal cells. Immunol Lett. 2014;159(12):55-72.

14. Tan KL, Scott DW, Hong F, et al. Tumorassociated macrophages predict inferior outcomes in classic Hodgkin lymphoma: a correlative study from the E2496 Intergroup trial. Blood. 2012;120(16):32803287.

15. Scott DW, Steidl C. The classical Hodgkin lymphoma tumor microenvironment: macrophages and gene expression-based modeling. Hematology Am Soc Hematol Educ Program. 2014;2014(1):144-150.

16. Ruella M, Klichinsky M, Kenderian SS, et al. Overcoming the immunosuppressive tumor microenvironment of Hodgkin lymphoma using chimeric antigen receptor $\mathrm{T}$ cells. Cancer Discov. 2017;7(10):1154-1167.

17. Tudor CS, Bruns H, Daniel C, et al. Macrophages and dendritic cells as actors in the immune reaction of classical Hodgkin lymphoma. PLoS One. 2014;9(12):e114345.

18. Koh YW, Park CS, Yoon DH, Suh C, Huh J. CD163 expression was associated with angiogenesis and shortened survival in patients with uniformly treated classical Hodgkin lymphoma. PLoS One. 2014; 9(1):e87066.

19. Crane GM, Samols MA, Morsberger LA, et al. Tumor-infiltrating macrophages in post- transplant, relapsed classical Hodgkin lymphoma are donor-derived. PLoS One. 2016;11(9):e0163559.

20. Tadmor T, Bari A, Marcheselli L, et al Absolute monocyte count and lymphocytemonocyte ratio predict outcome in nodular sclerosis Hodgkin lymphoma: evaluation based on data from 1450 patients. Mayo Clin Proc. 2015; 90(6):756-764.

21. Gotti M, Nicola $M$, Lucioni $M$, et al. Independent prognostic impact of tumourinfiltrating macrophages in early-stage Hodgkin's lymphoma. Hematol Oncol. 2017;35(3):296-302.

22. Carlo-Stella C, Santoro A Microenvironment-related biomarkers and novel targets in classical Hodgkin's lymphoma. Biomark Med. 2015;9(8):807-817.

23. Montanari F, Diefenbach CS. Hodgkin lymphoma: targeting the tumor microenvironment as a therapeutic strategy. Clin Adv Hematol Oncol. 2015;13(8):518-524.

24. Younes A, Ansell SM. Novel agents in the treatment of Hodgkin lymphoma: biological basis and clinical results. Semin Hematol. 2016;53(3):186-189.

25. von TB, Moskowitz CH. Treatment of relapsed and refractory Hodgkin lymphoma. Semin Hematol. 2016;53(3):180-185.

26. Weir SJ, DeGennaro LJ, Austin CP. Repurposing approved and abandoned drugs for the treatment and prevention of cancer through public-private partnership. Cancer Res. 2012;72(5):1055-1058.

27. Halama N, Zoernig I, Berthel A, et al. Tumoral immune cell exploitation in colorectal cancer metastases can be targeted effectively by Anti-CCR5 therapy in cancer patients. Cancer Cell. 2016;29(4):587-601.

28. Abel S, van der Ryst E, Rosario MC, et al Assessment of the pharmacokinetics, safety and tolerability of maraviroc, a novel CCR5 antagonist, in healthy volunteers. $\mathrm{Br}$ J Clin Pharmacol. 2008;65 Suppl 1:5-18.

29. Genebat M, Ruiz-Mateos E, Pulido I, et al Long-term immunovirogical effect and tol- 
erability of a maraviroc-containing regimen in routine clinical practice. Curr HIV Res. 2010;8(6):482-486.

30. Velasco-Velazquez M, Jiao X, De La Fuente $\mathrm{M}$, et al. CCR5 antagonist blocks metastasis of basal breast cancer cells. Cancer Res. 2012;72(15):3839-3850.

31. Halvorsen EC, Hamilton MJ, Young A, et al. Maraviroc decreases CCL8-mediated migration of CCR5(+) regulatory $\mathrm{T}$ cells and reduces metastatic tumor growth in the lungs. Oncoimmunology. 2016; 5(6):e1150398.

32. Tanabe Y, Sasaki S, Mukaida N, Baba T. Blockade of the chemokine receptor, CCR5, reduces the growth of orthotopically injected colon cancer cells via limiting cancer-associated fibroblast accumulation. Oncotarget. 2016;7(30):48335-48345.

33. Weiswald LB, Bellet D, Dangles-Marie V. Spherical cancer models in tumor biology. Neoplasia. 2015;17(1):1-15.
34. Mencarelli A, Graziosi L, Renga B, et al. CCR 5 antagonism by maraviroc reduces the potential for gastric cancer cell dissemination. Transl Oncol. 2013;6(6):784-793.

35. Lejmi E, Perriraz N, Clement $S$, et al Inflammatory chemokines MIP-1delta and MIP-3alpha are involved in the migration of multipotent mesenchymal stromal cells induced by hepatoma cells. Stem Cells Dev. 2015;24(10):1223-1235.

36. Poggi A, Zocchi MR. Stress immunity in lymphomas: mesenchymal cells as a target of therapy. Front Biosci (Landmark Ed). 2014;19:281-290.

37. Zocchi MR, Catellani S, Canevali $\mathrm{P}$, et al High ERp5/ADAM10 expression in lymph node microenvironment and impaired NKG2D ligands recognition in Hodgkin lymphomas. Blood. 2012;119(6):1479-1489.

38. Barros MH, Segges P, Vera-Lozada G, Hassan R, Niedobitek G. Macrophage polarization reflects $\mathrm{T}$ cell composition of tumor microenvironment in pediatric classical Hodgkin lymphoma and has impact on survival. PLoS One. 2015; 10(5):e0124531.

39. Dorsam B, Bosl T, Reiners KS, et al. Hodgkin lymphoma-derived extracellular vesicles change the secretome of fibroblasts toward a CAF phenotype. Front Immunol. 2018;9:1358.

40. Mantovani A, Marchesi F, Malesci A, Laghi L, Allavena P. Tumour-associated macrophages as treatment targets in oncology. Nat RevClin Oncol. 2017;14(7):399 416.

41. Carey CD, Gusenleitner D, Lipschitz M, et al. Topological analysis reveals a PD-L1associated microenvironmental niche for Reed-Sternberg cells in Hodgkin lymphoma. Blood. 2017;130(22):2420-2430.

42. Jiao X, Velasco-Velazquez MA, Wang M, et al. CCR5 governs DNA damage and breast cancer stem cell expansion. Cancer Res. 2018;78(7):1657-1671. 